\title{
STATE OF CUTANEOUS MICROCIRCULATION IN PATIENTS WITH OBESITY
}

DOI: 10.36740/WLek202109103

\author{
lana 0. Andreieva', Olha I. Riznyk' ${ }^{2}$, Sergii P. Myrnyi' ${ }^{2}$, Nikolai N. Surmylo ${ }^{1}$ \\ 'ZAPORIZHZHIA MEDICAL ACADEMY OF POST-GRADUATE EDUCATION MINISTRY OF HEALTH OF UKRAINE, ZAPORIZHZHIA, UKRAINE \\ ${ }^{2}$ ZAPORIZHZHIA STATE MEDICAL UNIVERSITY, ZAPORIZHZHIA, UKRAINE
}

\begin{abstract}
The aim: To determine the influence of obesity on cutaneous microcirculation in patients with different stages of obesity and without cardio-vascular pathologies. Materials and methods: The 67 eligible patients with obesity were enrolled into the main group in this research. 20 healthy patients with normal body weight were included in the control group in this study. The mean age and gender were similar among the groups. Each patient underwent a clinical evaluation during the consultation, biological tests, electrocardiogram, Laser Doppler flowmetry.

Results: There was a significant decrease in IM and $\sigma$ in patients with class II obesity and class III obesity compared with control group. IV was decreased in all groups, but the significant differences were recorded only among patients with obesity and not among overweight patients. During wavelet analysis a significant decrease of the AmaxE was detected in all 4 groups (by 14,7\%, 37,7\%,52,4\%,57,4\% respectively, $\mathrm{P}<0,05$ ). The most significant changes were recorded in the heart spectrum (AmaxC).

Conclusions: Overweight patients and patients with obesity without cardio-vascular pathology have initial manifestations of microcirculatory disorders, which deteriorate with an increase of BMI. Changes in microcirculation in patients with obesity characterized by the significant decrease of microvessels perfusion, decreasing endothelial and increasing heart specter modulations. Only high BMI was found to be associated with impaired microcirculation endothelial function (AmaxE B=0.446, 95\% Cl [0,15, 0,92]) according to the results of regression analysis.
\end{abstract}

KEY WORDS: obesity, microcirculation, overweight

Wiad Lek. 2021;74(9 p.l):2039-2043

\section{INTRODUCTION}

The prevalence of obesity has nearly doubled worldwide during the past three decades, and it is still increasing [1]. Obesity is associated with several major cardiovascular risk factors, such as the metabolic syndrome, arterial hypertension $(\mathrm{AH})$, and type 2 diabetes mellitus, dyslipidemia, which lead to increased cardiovascular and all-cause mortality in obese people [2]. Nevertheless, numerous studies have documented an obesity paradox, in which overweight and obese patients demonstrate a better prognosis compared with lean or underweight heart failure patients in comparison with the other cardiovascular diseases [3]. The pathogenic explanation of obesity paradox is still obscure. The importance of microvascular vasomotor dysfunction in contributing to the morbidity and mortality of obese patients was appreciated many years ago and some authors consider it as lost pathogenic link in obesity paradox $[3,4]$.

Obesity has detrimental effects on the microcirculation. Functional changes in microvascular responsiveness may increase the risk of developing cardiovascular complications in obese patients $[1,5]$. Some observational studies [2, $5,6]$ provide evidence that obesity, having any co-existing cardiovascular diseases, is associated with the impaired vasomotor function of arteries, veins, and microvessels. At the same time, it was shown that a lower BMI appears to be a primary determinant of microvascular resistance [7]. Higher BMI can lead to microvascular dilatation and improve microvascular reactivity $[8,9]$. According to these statements, features of microvascular reaction in patients with obesity could be considered as main pathogenetic mechanisms and examination of microcirculation in patients with obesity could help to explain obesity paradox.

Nowadays the cutaneous microcirculation has emerged as a clinically relevant model for microvascular reactivity and endothelial function. Laser Doppler flowmetry (LDF) is frequently used to measure changes in blood cell flow (flux) in the skin microcirculation after stimulation. Different stimuli are used for probing microcirculation function, and different methods are used for analyzing the vascular response. Several studies have been published, providing factors influencing endothelial and microvascular function. However, the results vary significantly, possibly because of different population study.

\section{THE AIM}

The aim of the study was to determine the influence of obesity on cutaneous microcirculation in patients with different stages of obesity and without cardio-vascular pathologies. 


\section{MATERIALS AND METHODS}

The observational study was conducted in the therapeutical department of the CU "Central hospital of Komunarskyi district" from May, 2017 to June, 2019. The sixty-seven eligible patients with obesity were enrolled into the main group in this research. Twenty healthy subjects with normal body weight were included into the control group in this study. The mean age and gender were similar among the groups.

Our present research was approved by the clinical research ethics committee of SI "Zaporizhzhia Medical Academy of Post-Graduate Education Ministry of Health of Ukraine". Written informed consent was obtained from all patients. This research was conducted in accordance with "the 1964 Helsinki Declaration."

Exclusion criteria from the study were the following: patients with known cardiovascular pathologies, respiratory pathologies, diabetes or thyroid dysfunction, any known renal diseases, cognitive impairment, dementia, drug and alcohol abuse, skin diseases, rejection to sign the informed consents.

Each patient underwent a clinical evaluation during the consultation (with measurement of body mass and height), biological tests, electrocardiogram (Cardio Sens, XAI-Medica, Ukraine), Laser Doppler flowmetry (LDF) (SPE “LAZMA” Ltd., Russia Federation).

Body mass index (BMI) of the patients was calculated as weight divided by height squared $\left(\mathrm{kg} \cdot \mathrm{m}^{2}\right)$. According to BMI, all subjects were divided into four groups. The first group (group I) included participants who were overweight $(25,0-29,9)(26$ patients); the second group (group II)- I class obesity (BMI - 30 - 34,9 kg.m2) (25 patients); the third group (group III) - with II class obesity (BMI - 35- 39,9 $\mathrm{kg} \cdot \mathrm{m} 2$ ) (10 patients); the fourth group (group IV) - with III class obesity (BMI $\geq 40,0 \mathrm{~kg} \cdot \mathrm{m} 2$ ) (6 patients). Clinical characteristic of enrolled patients is shown on table I.
The Laser Doppler probe was applied at the dorsum of the hand, avoiding any underlying bony structures or large vessels. All recordings were taken from the palmar skin surface of the distal phalanx of the third finger. We evaluated the mean index of microcirculation (IM), index of variation $\left(I_{v}\right)$, the standard deviation of IM $(\sigma)$. With the wavelet analysis, we studied the maximum amplitude of blood flow oscillations with different specters: active frequency ranges (endothelial activity (AmaxE) (0,0095$0,02 \mathrm{~Hz})$; neurogenic activity $($ AmaxH) $(0,02-0,046 \mathrm{~Hz})$; myogenic activity (AmaxM) (0,07-0,145 Hz)) and passive frequency ranges (cardiac or heart ranges (AmaxC) (0,8-1,6 $\mathrm{Hz})$, respiratory ranges $(\mathrm{AmaxD})(0,2-0,4 \mathrm{~Hz}))$. Microcirculation investigations were carried out at the baseline state and after arterial occlusion tests. The blood pressure and the LDF-gram were recorded within 10 minutes after the end of hemorrhage (post hemorrhagic period).

Statistical analysis. The quantitative variables were expressed as means \pm SD. Categorical variables were presented as percentages. The differences in each variable were evaluated by the Student's t-test for continuous variables and the $\chi 2$ test for categorical variables. The relationships between parameters were evaluated by Pearson correlation analysis and univariate regression analysis. A P-value < 0,05 was considered as an indication statistically significant difference between groups. Calculations were performed with SPSS-software (Version 23.0; SPSS, Chicago, IL).

\section{RESULTS}

Microcirculation parameters were compared with the groups. According to the results of our study, there was a significant decrease in IM and $\sigma$ in patients with class II obesity and class III obesity compared with control group (table II).

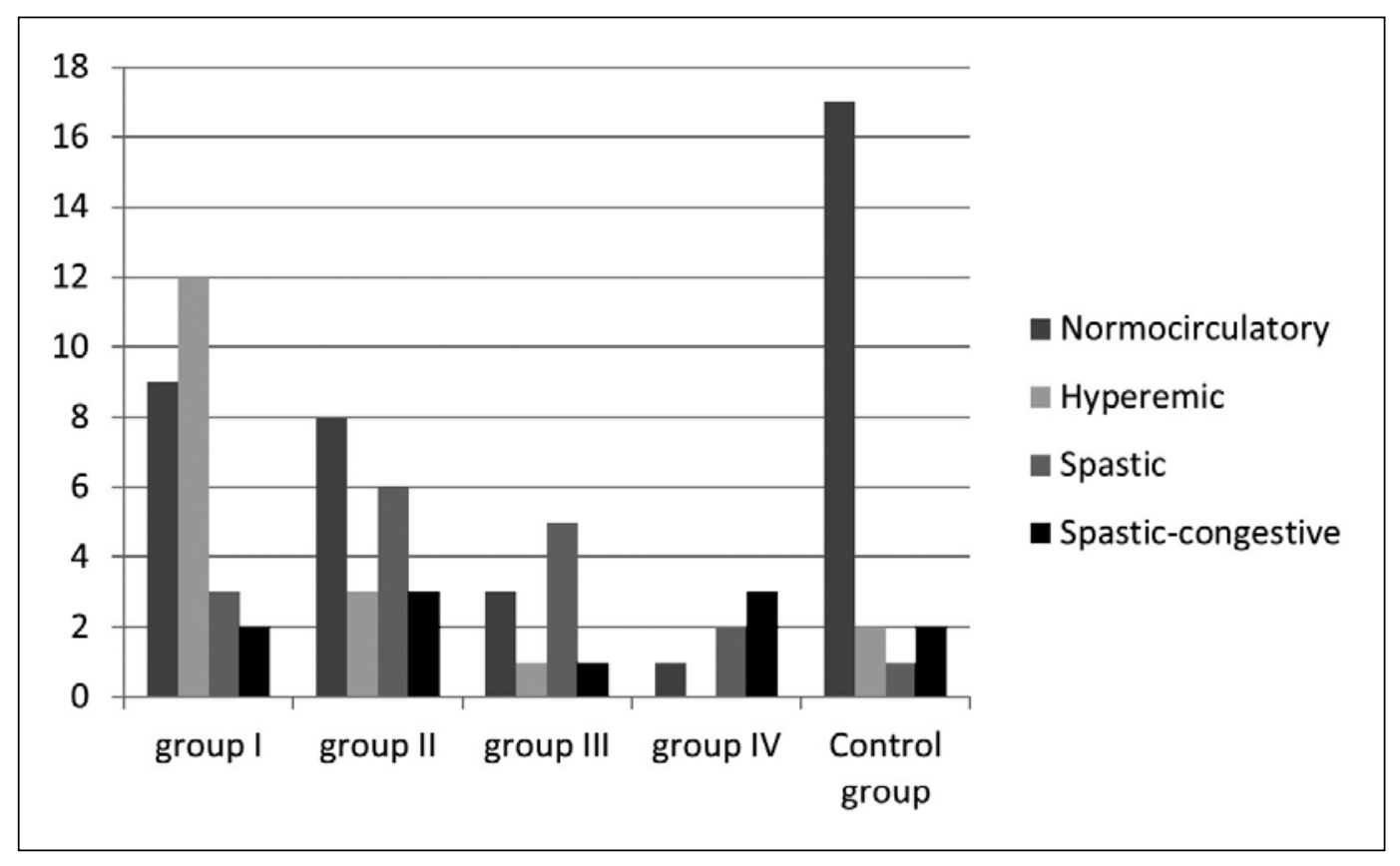

Fig 1. Distribution of enrolled patients according to microcirculation types 
Table I. Clinical characteristic of enrolled patients

\begin{tabular}{|c|c|c|c|c|c|}
\hline Parameters, units & $\begin{array}{l}\text { Group I } \\
(n=26)\end{array}$ & $\begin{array}{l}\text { Group II } \\
(n=25)\end{array}$ & $\begin{array}{l}\text { Group III } \\
(n=10)\end{array}$ & $\begin{array}{c}\text { Group IV } \\
(n=6)\end{array}$ & $\begin{array}{c}\text { Control group } \\
(n=20)\end{array}$ \\
\hline Male/Female (n) & $14 / 12$ & $15 / 10$ & $4 / 6$ & $2 / 4$ & $12 / 8$ \\
\hline Mean age (years) & $36,5 \pm 6,8$ & $37,7 \pm 5,2$ & $39,2 \pm 6,2$ & $39,6 \pm 6,1$ & \multirow{2}{*}{$38,5 \pm 3,6$} \\
\hline$P_{\text {control }}$ & 0,122 & 0,095 & 0,021 & 0,071 & \\
\hline BMI $\left(\mathrm{kg} / \mathrm{m}^{2}\right)$ & $27,2 \pm 3,3$ & $32,9 \pm 2,6$ & $37,5 \pm 4,6$ & $42,6 \pm 2,2$ & \multirow{2}{*}{$22,1 \pm 3,3$} \\
\hline$P_{\text {control }}$ & 0,023 & 0,011 & 0,005 & 0,005 & \\
\hline Waist circumference, $\mathrm{cm}$ & $82,32 \pm 4,15$ & $90,41 \pm 4,08$ & $107,64 \pm 3,11$ & $115,80 \pm 3,66$ & \multirow{2}{*}{$76,91 \pm 6,24$} \\
\hline $\mathrm{P}_{\text {control }}$ & 0,034 & 0,028 & 0,001 & 0,003 & \\
\hline Hip circumference, cm & $98,71 \pm 2,88$ & $102,28 \pm 2,13$ & $112,19 \pm 3,04$ & $119,32 \pm 2,79$ & \multirow{2}{*}{$93,61 \pm 3,44$} \\
\hline $\mathrm{P}_{\text {control }}$ & 0,023 & 0,035 & 0,001 & 0,001 & \\
\hline Waist-hip ratio, y.o. & $0,78 \pm 0,03$ & $0,82 \pm 0,02$ & $0,87 \pm 0,03$ & $0,92 \pm 0,03$ & \multirow{2}{*}{$0,71 \pm 0,08$} \\
\hline$P_{\text {control }}$ & 0,056 & 0,015 & 0,028 & 0,003 & \\
\hline Duration of obesity, years & $10,2 \pm 3,5$ & $12,8 \pm 5,1$ & $14,8 \pm 5,5$ & $16,2 \pm 2,6$ & 0 \\
\hline
\end{tabular}

Table II. Microcirculation parameters in enrolled patients

\begin{tabular}{|c|c|c|c|c|c|}
\hline Parameters, units & $\begin{array}{l}\text { Control group } \\
(\mathbf{n = 2 0 )}\end{array}$ & $\begin{array}{l}\text { Group I } \\
(n=26)\end{array}$ & $\begin{array}{l}\text { Group II } \\
(n=25)\end{array}$ & $\begin{array}{l}\text { Group III } \\
(n=10)\end{array}$ & $\begin{array}{c}\text { Group IV } \\
(n=6)\end{array}$ \\
\hline $\mathrm{IM}$ & \multirow{2}{*}{$16,72 \pm 3,44$} & $17,22 \pm 1,27$ & $14,11 \pm 2,15$ & $13,09 \pm 1,78$ & $15,45 \pm 1,59$ \\
\hline$P_{\text {control }}$ & & 0,1018 & 0,845 & 0,042 & 0,103 \\
\hline$\sigma$ & \multirow{2}{*}{$2,33[1,97 ; 2,88]$} & $2,13[1,93 ; 2,91]$ & $1,85[1,36 ; 2,68]$ & $1,59[1,14 ; 2,58]$ & $1,97[1,13 ; 2,74]$ \\
\hline$P_{\text {control }}$ & & 0,912 & 0,121 & 0,022 & 0,001 \\
\hline $\mathrm{I}_{\mathrm{V}}$ & \multirow{2}{*}{$14,22 \pm 2,67$} & $13,19 \pm 1,79$ & $11,68 \pm 2,01$ & $9,03 \pm 1,79$ & $8,72 \pm 1,74$ \\
\hline$P_{\text {control }}$ & & 0,157 & 0,006 & 0,001 & 0,001 \\
\hline AmaxE & \multirow{2}{*}{$0,61 \pm 0,05$} & $0,42 \pm 0,05$ & $0,38 \pm 0,04$ & $0,29 \pm 0,04$ & $0,26 \pm 0,03$ \\
\hline$P_{\text {control }}$ & & 0,012 & 0,003 & 0,001 & 0,001 \\
\hline AmaxH & \multirow{2}{*}{$0,64 \pm 0,05$} & $0,53 \pm 0,06$ & $0,68 \pm 0,04$ & $0,72 \pm 0,04$ & $0,76 \pm 0,05$ \\
\hline$P_{\text {control }}$ & & 0,052 & 0,004 & 0,001 & 0,001 \\
\hline AmaxM & \multirow{2}{*}{$0,48 \pm 0,04$} & $0,38 \pm 0,04$ & $0,33 \pm 0,03$ & $0,29 \pm 0,03$ & $0,22 \pm 0,03$ \\
\hline$P_{\text {control }}$ & & 0,003 & 0,003 & 0,001 & 0,001 \\
\hline AmaxD & \multirow{2}{*}{$0,27 \pm 0,06$} & $0,29 \pm 0,03$ & $0,34 \pm 0,02$ & $0,37 \pm 0,04$ & $0,46 \pm 0,02$ \\
\hline$P_{\text {control }}$ & & 0,074 & 0,007 & 0,016 & 0,002 \\
\hline \multirow{2}{*}{ AmaxC } & \multirow{2}{*}{$0,38 \pm 0,06$} & $0,49 \pm 0,05$ & $0,52 \pm 0,04$ & $0,58 \pm 0,03$ & $0,61 \pm 0,06$ \\
\hline & & 0,044 & 0,006 & 0,002 & 0,001 \\
\hline
\end{tabular}

IM was significantly lower in patients from group III $\left(P_{k}=0,042\right)$ and from group IV $\left(P_{k}=0,103\right)$ compared to control group. Overweight patients didn 't show statistically significant increase of IM in comparison with control group.

$\mathrm{I}_{\mathrm{v}}$ was decreased in all groups, but the significant differences were recorded only among patients with obesity and not among overweight patients. In addition, there was a significant decrease in IEM in all three groups of obese patients. The IEM changes were not statistically significant among overweight patients.

During wavelet analysis a significant decrease of the AmaxE was detected in all four groups (by $14,7 \%, 37,7 \%$, $52,4 \%, 57,4 \%$ respectively, $\mathrm{P}<0,05)$. Also, AmaxN and AmaxM were decreased among patients with obesity in comparison with overweight and control groups. Among passive frequency ranges, a significant increase in AmaxR was recorded in all three groups of obese patients compared with the control group (by 25,9\%,37,04\%, and 44,4\% respectively), $(\mathrm{P}<0,05)$. The most significant changes were recorded in the heart spectrum (AmaxC). The AmaxC significantly exceeded the control group's results by $28,9 \%$ in group I, by $36,8 \%$ in group II, by $42,2 \%$ in group III and by $60,5 \%$ in group IV $(\mathrm{P}<0,05)$.

According to the results of occlusion test, the reserve of microcirculatory blood flow (RMB) was calculated and the type of microcirculation was estimated. The distribution of patients in groups depending on the type of microcirculation is shown in the figure 1 .

Hyperemic type of microcirculation is prevalent among patients with overweight. The frequency of spastic micro- 
circulation increased among patients with I class obesity, but this type of microcirculation was also prevalent among patients with II class obesity. The spastic-congestive type of microcirculation was predominant in patients with severe obesity (III class).

There was a significant correlation between $\mathrm{BMI}$ and $\mathrm{I}_{\mathrm{v}}$ $(r=-0,315, P=0,015)$ and AmaxE $(r=-0.411, P=0,003)$. IM is significantly correlated with waist-hip ratio $(r=-0,269, P=0,033)$, duration of hypertension $(r=-0.411, P=0,003)$, and smoking $(r=-0,316, P=0,034)$. Age is significantly correlated with $\operatorname{AmaxE}(r=-0,345, P=0,002)$, IM $(r=-0,218, P=0,031)$, AmaxH $(r=-0,218, P=0,031)$.

Using all predefined risk factors in selection (sex, age, smoking, hypertension, and BMI) in a linear multivariate analysis, only high BMI was found to be associated with impaired of microcirculation endothelial function (AmaxE B=0.446, 95\% CI $[0,15,0,92], \mathrm{P}=0,005$ ). When all risk factors were combined into a multivariable linear regression model, BMI still remained independently related to AmaxE.

\section{DISCUSSION}

According to our research, it has been found that overweight patients and patients with obesity have already initial manifestations of microcirculatory disorders, which deteriorate with an increase of BMI. There is deterioration of microcirculation in overweight and obesity patients even without cardio-vascular pathology. So, we can assume, that microvascular dysfunction potentially indicates a very early stage of vascular dysfunction and it might be the first sign of cardiovascular risk even before the appearance of classical metabolic risk factors. These changes include formation of local spasm among overweight patients and patients with I class obesity which progress to the decrease of vessel's tonus, the onset of stasis in arterioles and venues and the general deterioration in blood flow among patients with severe obesity. A significant impairment of the hyperemic flow response was showed in our study among patients with a high BMI. Reduced IM among obese patients indicates a reduction of tissue perfusion. At the same time, we have found relative increase of IM in patients with severe obesity, which may indicate a decrease in the tone of arterial vessels and the onset of blood stasis in the venues. The decrease of the $\sigma$ index, which is considered as an indicator of the temporary variability of perfusion, which usually indicates the inhibition of active vasomotor mechanisms of tissue flow modulation or about prevails of sympathetic nervous system influences.

The relative increase in the $\sigma$ index among patients with III class obesity may indicate the presence of stagnant phenomena in venues, which reduces perfusion pressure in the capillaries. This mechanism leads to an increase in the amplitude of the respiratory rhythm, which confirmed by the results of the amplitude-frequency analysis in our study. There was a significant increase in the amplitude of neurogenic modulation, which indicates an increase of impact of this mechanism of regulation, namely, the increase in the activity of the sympathetic nervous system. Experimental investigations have shown that with excess adiposity, in particular when visceral fat accumulation is present, sympathetic activation commonly occurs [10, 11]. The progressive decrease in the $\sigma$ index indicates a progressive deterioration of the microcirculation. Among the active mechanisms of regulation of microcirculation in patients with obesity vascular mechanism predominated.

Changes in microvascular hyperemic response were proved by some researches. In Denga O. study, obese patients had a significant lower microvascular hyperemic response to post occlusive reactive hyperemia compared with a healthy control population [12]. This difference diminished after lowering the BMI by gastric bypass surgery, both among patients with and without type 2 diabetes mellitus. In our study, we detected the same changes, but we discovered changes of cutaneous microcirculation and didn ' t observed patients after bariatric surgery. In line with these results, Dirk J. van der Heijden et al. study showed that a high BMI is an independent predictor of reduced microvascular endothelial function among patients with suspected and established vascular disease [5]. The authors have found that the relationship between BMI and microvascular endothelial dysfunction remains, even taking into account successfully treated obesity-related cardio metabolic risk factors such as hypertension, hypercholesterolemia, and diabetes mellitus.

Similar changes were shown in experimental works. For example, in study of Meijer R.I. et al. [13] and Aghamohammadzadeh R. et al. [14] it was shown that under normal circumstances, perivascular adipose tissue functions directly reducing smooth muscle tone and enhancing insulin-dependent vasodilatation. In obese mice, perivascular adipose tissue-dependent vascular relaxation is attenuated, partly as a results of reduced nitric oxide bioavailability. In our study, we have got the proof of local spasm of microcirculation vessels and progressive deterioration of vessels tonus in patients with increasing of BMI.

Limitations of our study should also be considered. We studied vascular function in the digits, which is only partly dependent on nitric oxide bioavailability, and the evaluation in different vascular beds may provide complementary information. However, microvascular function, measured by occlusion test, has been shown to be more closely correlated to metabolic risk factors than conduit vessel function and has been prospectively validated as a predictor of cardiovascular events. Secondly, the temperature of the skin is highly dependent on skin capillary blood volume and it might rather reflect the exchange capacity of the superficially located capillary endothelium than blood flow in deeper situated arterioles as detected by LDF. Third, other variables such as fat distribution and fat mass could add to our interpretation of the relation of adiposity to vascular function. Lastly, due to the observational design of the cohorts, endothelium-independent vasodilatation was not evaluated and the use of medications could not be precluded. Since our analysis is cross-sectional, it prevents inferences of causality or temporality. 


\section{CONCLUSIONS}

Overweight patients and patients with obesity without cardio-vascular pathology have initial manifestations of microcirculatory disorders, which deteriorate with an increase of BMI. Changes in microcirculation in patients with obesity characterized by the significant decrease of microvessels perfusion (decreasing of IM, $\sigma$, and $\mathrm{K}_{\mathrm{v}}$ ), decreasing endothelial and increasing heart specter modulations. Only high BMI was found to be associated with impaired microcirculation endothelial function (AmaxE $\mathrm{B}=0.446,95 \% \mathrm{CI}[0,15,0,92])$ according to the results of regression analysis.

\section{REFERENCES}

1. Blüher M. Obesity: global epidemiology and pathogenesis. Nature Reviews Endocrinology. 2019;15(5):288-298.

2. Andolfi C., Fisichella P. Epidemiology of Obesity and Associated Comorbidities. Journal of Laparoendoscopic \& Advanced Surgical Techniques. 2018;28(8):919-924.

3. Elagizi A., Kachur S., Lavie C. et al. An Overview and Update on Obesity and the Obesity Paradox in Cardiovascular Diseases. Progress in Cardiovascular Diseases. 2018;61(2):142-150.

4. Horwich T, Fonarow G, Clark A. Obesity and the Obesity Paradox in Heart Failure. Progress in Cardiovascular Diseases. 2018;61(2):151-156.

5. Van der Heijden D., van Leeuwen M., Janssens G. et al. Body Mass Index Is Associated With Microvascular Endothelial Dysfunction in Patients With Treated Metabolic Risk Factors and Suspected Coronary Artery Disease. Journal of the American Heart Association. 2017;6(9):e006082.

6. Meijer R., SernéE., Korkmaz H. et al. nsulin-induced changes in skeletal muscle microvascular perfusion are dependent upon perivascular adipose tissue in women. Diabetologia.2015;58(8):1907-1915.

7. Bradley E., Richards S., Keske M., Rattigan S. Local NOS inhibition impairs vascular and metabolic actions of insulin in rat hindleg muscle in vivo. American Journal of Physiology-Endocrinology And Metabolism.2013;305(6):E745-E750.

8. Nernpermpisooth N., Qiu S., Mintz J. et al.. Obesity Alters the Peripheral Circadian Clock in the Aorta and Microcirculation. Microcirculation.2015;22(4): 257-266.

9. Montero D., Walther G., Perez-Martin A. et al. Effects of a Lifestyle Program on Vascular Reactivity in Macro- and Microcirculation in Severely Obese Adolescents. The Journal Of Clinical Endocrinology \& Metabolism. 2014; 99(3):1019-1026.
10. Harrell J., Johansson R., Evans T. et al. Preserved Microvascular Endothelial Function in Young, Obese Adults with Functional Loss of Nitric Oxide Signaling. Frontiers in Physiology. 2015;6.

11. Frisbee J. Obesity, insulin resistance, and microvascular adaptation. Microcirculation. 2017;24(2):e12346.

12. Denga 0., Pyndus T., Gargin V., Schneider S. Influence of metabolic syndrome on condition of microcirculatory bed of oral cavity. Georgian Med News. 2017;273:99-104.

13. Meijer R., Bakker W., Alta C., Sipkema P. Perivascular Adipose Tissue Control of Insulin-Induced Vasoreactivity in Muscle Is Impaired in db/ db Mice. Diabetes. 2012;62(2):590-598.

14. Aghamohammadzadeh R., Unwin R., Greenstein A., Heagerty A. Effects of Obesity on Perivascular Adipose Tissue Vasorelaxant Function: Nitric Oxide, Inflammation and Elevated Systemic Blood Pressure. Journal of Vascular Research.2015;52(5):299-305.

\section{ORCID and contributionship:}

Iana O. Andreieva: 0000-0002-7959-992X $X^{A, C, F}$

Olha I. Riznyk: 0000-0002-2658-2120 ${ }^{B, D}$

Sergii P. Myrnyi: 0000-0002-0789-7282 B,E

Nikolai N. Surmylo: 0000-0003-2333-1892 ${ }^{D, E}$

\section{Conflict of interest:}

The Authors declare no conflict of interest.

\section{CORRESPONDING AUTHOR lana 0. Andreieva}

Zaporizhzhia Medical Academy of Post-Graduate

Education Ministry of Health of Ukraine

20 Winter Boulevard, 69000 Zaporizhzhia, Ukraine

tel: +380950650330

e-mail:andryana08@gmail.com

Received: 17.08 .2020

Accepted: 02.06.2021

A - Work concept and design, B - Data collection and analysis, C - Responsibility for statistical analysis,

D-Writing the article, $\mathbf{E}$ - Critical review, $\mathbf{F}$ - Final approval of the article 